\title{
Modeling Mixed Bicycle Traffic Flow: A Comparative Study on the Cellular Automata Approach
}

\author{
Dan Zhou, Sheng Jin, Dongfang Ma, and Dianhai Wang \\ College of Civil Engineering and Architecture, Zhejiang University, Hangzhou 310058, China \\ Correspondence should be addressed to Sheng Jin; jinsheng@zju.edu.cn
}

Received 10 December 2014; Revised 29 April 2015; Accepted 30 April 2015

Academic Editor: Tetsuji Tokihiro

Copyright (C) 2015 Dan Zhou et al. This is an open access article distributed under the Creative Commons Attribution License, which permits unrestricted use, distribution, and reproduction in any medium, provided the original work is properly cited.

\begin{abstract}
Simulation, as a powerful tool for evaluating transportation systems, has been widely used in transportation planning, management, and operations. Most of the simulation models are focused on motorized vehicles, and the modeling of nonmotorized vehicles is ignored. The cellular automata (CA) model is a very important simulation approach and is widely used for motorized vehicle traffic. The Nagel-Schreckenberg (NS) CA model and the multivalue CA (M-CA) model are two categories of CA model that have been used in previous studies on bicycle traffic flow. This paper improves on these two CA models and also compares their characteristics. It introduces a two-lane NS CA model and M-CA model for both regular bicycles (RBs) and electric bicycles (EBs). In the research for this paper, many cases, featuring different values for the slowing down probability, lane-changing probability, and proportion of EBs, were simulated, while the fundamental diagrams and capacities of the proposed models were analyzed and compared between the two models. Field data were collected for the evaluation of the two models. The results show that the M-CA model exhibits more stable performance than the two-lane NS model and provides results that are closer to real bicycle traffic.
\end{abstract}

\section{Introduction}

Traffic flow theories are generally divided into two branches: macroscopic and microscopic theories [1]. The macroscopic traffic flow models are based on fluid dynamics and are mostly used to elucidate the relationships between density, volume, and speed (also called the fundamental diagram) in various traffic conditions. The microscopic traffic models, on the other hand, describe the interaction between individual vehicles. The microscopic traffic models generally include car-following models and cellular automata (CA) models. The car-following model is the most important model, describing the detailed movements of vehicles proceeding close together in a single lane. There have been many carfollowing models produced in the literature over the past 60 years, such as stimulus-response models, safety distance models, action point models, fuzzy-logic-based models, and optimal velocity models [2-5]. For a broader review, refer to Brackstone and McDonald [6] and Chowdhury et al. [7]. Recently, CA models have emerged as an efficient tool for simulating highway traffic flow because of their easy concept, simple rule, and speed in conducting numerical investigations. The rule-184 model, proposed by Wolfram [8], was the first CA model to be widely used for traffic flow. Nagel and Schreckenberg [9] presented the well-known NS CA model, which is an extension of the rule-184 model allowing the maximal speed of vehicles to be more than one cell/s. The NS model and the many improved versions of it reproduce some basic and complicated phenomena such as stop and go, metastable states, capacity drop phenomena (which means the capacity of road experiences a large drop under critical density conditions), and synchronized flow in real traffic conditions.

Most of the aforementioned microscopic traffic models have been developed only for motorized vehicles. Few of them have been used for modeling non-motorized vehicles such as bicycles, tricycles, electric bicycles, and motorcycles because of the complicated characteristics of such vehicles movements. With the increasing usage of bicycles, some researchers have begun focusing on modeling the operation of bicycle facilities. Jiang et al. [10] introduced two different multivalue CA (M-CA) models in order to model bicycle 
flow. Their simulation results showed that, once the randomization effect is considered, the multiple states in deterministic M-CA models disappear and unique flow-density relations exist. They found the transition from free flow to congested flow to be smooth in one model but of second order in the other. Lan and Chang [11] developed inhomogeneous CA models to elucidate the interacting movements of cars and motorcycles in mixed traffic contexts. The car and motorcycle were represented by nonidentical particle sizes, respectively, occupying $6 \times 2$ and $2 \times 1$ cell units, each of size $1.25 \times 1.25$ meters. The CA models were validated by a set of field-observed data and the relationships between flow, cell occupancy (a proxy of density), and speeds under different traffic mixtures and road (lane) widths were elaborated. A M-CA model for mixed bicycle flow was proposed by Jia et al. [12]. Two types of bicycles, with different maximum speeds $(1$ cell/s and 2 cells/s), were considered in the system. Different results were analyzed and investigated under both deterministic and stochastic regimes. Li et al. [13] presented a multivalue cellular model for mixed nonmotorized traffic flow composed of bicycles and tricycles. A bicycle was assumed to occupy one unit of cell space and a tricycle two units of cell space. The simulation results showed the multiple state effect of mixed traffic flow. Gould and Karner [14] proposed a two-lane inhomogeneous CA simulation model, an improved version of the NS model combining a lanechanging rule, for bicycle traffic, and collected field data from three UC Davis bike paths for comparison with a simulation model. Yang et al. [15] proposed an extended multivalue CA model that permitted the bicycles to move at faster speeds. The simulation results showed that the mixed nonmotorized traffic capacity increased with an increase in the electric bicycle ratio. Zhang et al. [16] used an improved three-lane NS model to analyze the speed-density characteristics of mixed bicycle flow. The simulation results of the CA model were effectively consistent with the actual survey data when the density was lower than $0.225 \mathrm{bic} / \mathrm{m}^{2}$.

Summarizing the above, none of the aforementioned carfollowing models have been devoted to mixed traffic with regular bicycles (RBs) and electric bicycles (EBs), but CA models have been widely used for modeling nonmotorized traffic. The modeling of mixed bicycle traffic using CA models can be divided into two branches: NS CA model and M-CA model approaches. The update rules of the NS CA model for bicycle flow are the same as for motorized vehicles, with only the cell size and bicycle speeds being different. As pointed out by Jiang et al. [10] and Jia et al. [12], the M-CA model is more suitable than the NS model for modeling bicycle traffic flow. Because the update rules of the M-CA model do not include direct car-following and lane-changing behavior, it may be appropriate for modeling the nonlane-based behavior of bicycle traffic. The NS CA model and M-CA model have been used for modeling bicycle traffic and mixed bicycle traffic with RBs and EBs. However, there is no evidence in the existing literature as to which model is better for modeling mixed traffic flow, nor as to the differences between these two models. Therefore, a comparison of the NS CA model and the M-CA model in terms of their ability to model mixed bicycle traffic is required so that CA models can be improved efficiently.

This paper attempts to develop two CA models to describe the behaviors of mixed bicycle traffic with RBs and EBs on a separated bicycle path and to compare the characteristics of the NS CA model and the M-CA model. The remaining parts are organized as follows. Section 2 introduces the development of NS and M-CA rules. Section 3 presents the simulation results of these two CA models. Section 4 further discusses differences in the simulation results. Finally, the conclusions and ideas for future studies are addressed.

\section{CA Models}

2.1. Definition of Cell Size and Bicycle Speed. The main differences encountered when modeling bicycle traffic as opposed to motorized vehicle traffic using a CA model are the cell size and the speed. Mixed bicycle traffic with RBs and EBs on separated bicycle paths is ubiquitous in many Asian countries, such as China, Vietnam, Indonesia, and Malaysia. Because of the different operating speeds of RBs and EBs, mixed traffic produces complicated behavior and characteristics that are likely to lead to safety and efficiency problems. Modeling mixed bicycle traffic is very important for the planning, operation, and management of bicycle facilities. Based on the behavior of cyclists, CA models are the best option for modeling bicycle traffic. The size of cell space and the update rules are two significant aspects of CA models.

Bicycles are shorter and narrower than motorized vehicles. Based on field surveys, the length of most RBs and EBs is $1.7-1.9 \mathrm{~m}$. Meanwhile, bicycle lanes are set at 1 meter wide in both China and the USA $[17,18]$. Therefore, the size of a RB or an EB is assumed rectangular, with length $2 \mathrm{~m}$ and width $1 \mathrm{~m}$, as is widely used in other CA models [14-16]. The other parameter for modeling bicycle traffic is speed. According to the literature, the reported free flow speed of EBs is larger than that of RBs. Accordingly, in this paper speeds of 2 cells/s $(4 \mathrm{~m} / \mathrm{s}$ or $14.4 \mathrm{~km} / \mathrm{h})$ and 3 cells $/ \mathrm{s}(6 \mathrm{~m} / \mathrm{s}$ or $21.6 \mathrm{~km} / \mathrm{h})$ were chosen for RBs and EBs, respectively.

2.2. NS CA Model. The NS CA model used in this paper was proposed by Nagel and Schreckenberg [9]. This model is very widely used in modeling highway traffic and bicycle traffic. The NS CA model includes a car-following rule and a lane-changing rule. The car-following rule is based on four steps, and the lane-changing rule is based on the work of Rickert et al. [19]. Different vehicle behavior rules would lead to different simulation results. With an increase in the number of lanes, the lane-changing logic would become more complicated and make modeling more difficult. Therefore, in this paper only a two-lane bicycle path is simulated and used in the comparison. In the time interval from $t$ to $t+1$, the four basic rules of the NS model evolve according to the following steps.

Step 1 (longitudinal acceleration). Consider

$$
v_{i}(t+1)=\min \left(v_{i}(t)+1, v_{i \max }\right),
$$


where $v_{i}(t)$ is the speed of the $i$ th bicycle at updating time $t$. $v_{i \max }$ is the maximum speed of the $i$ th bicycle. This corresponds to the cyclists' realistic free flow speed.

Step 2 (longitudinal deceleration). Consider

$$
v_{i}(t+1)=\min \left(v_{i}(t), \operatorname{gap}_{i}\right),
$$

where gap $_{i}$ is the distance between the $i$ th bicycle and the bicycle in front of it, at updating time $t$. This step ensures that the bicycle stays safe with no collisions.

Step 3 (random slowing down). Consider

$$
v_{i}(t+1)=\max \left(v_{i}(t)-1,0\right) \text { if } \operatorname{rand}()<p_{d i},
$$

where $\operatorname{rand}()$ is a uniformly distributed random number between 0 and 1 and $p_{d i}$ is the random slowing down probability of the $i$ th bicycle. The random slowing down effect, which captures one cyclist's braking maneuver due to a random event (e.g., accident, road, or weather related factors), is one of the most significant parameters of the CA model. This step incorporates the idea of random effects on bicycles that may cause them to slow down.

Step 4 (motion). Consider

$$
x_{i}(t+1)=x_{i}(t)+v_{i}(t+1),
$$

where $x_{i}(t)$ is the position of the $i$ th bicycle at time $t$.

The lane-changing logic is shown below. Before the acceleration step, both lanes are examined to evaluate lane-changing opportunities. The following conditions are checked for each bicycle and must be true in order for it to change lanes.

(1) The speed of the bicycle currently in $i$ th position is larger than or equal to the cell distance to the next bicycle. This condition ensures that this bicycle will need to slow down at the next update:

$$
v_{i}(t) \geq \operatorname{gap}_{i}(t)
$$

(2) The distance to the next bicycle in the lane adjacent to the lane of the $i$ th bicycle $\left.\operatorname{gap}_{i}^{f}(t)\right)$ is larger than the distance to the next bicycle in its current lane $\left(\operatorname{gap}_{i}(t)\right)$. This condition ensures that a benefit is derived from changing lanes:

$$
\operatorname{gap}_{i}^{f}(t)>\operatorname{gap}_{i}(t)
$$

(3) The distance to backward bicycle in the lane adjacent to that of the currently ith bicycle $\left(\operatorname{gap}_{i}^{b}(t)\right)$ is large enough. This condition ensures that, looking backwards, the closest bicycle in the adjacent lane is sufficiently far away:

$$
\operatorname{gap}_{i}^{b}(t) \geq \min \left[v_{i-1}^{b}(t)+1, v_{i-1 \max }^{b}\right] .
$$

(4) A uniformly distributed random number between 0 and 1 is less than the probability of a lane change $\left(p_{t}\right)$ :

$$
\text { rand }()<p_{t} \text {. }
$$

$\operatorname{gap}_{i}^{f}(t)$ and $\operatorname{gap}_{i}^{b}(t)$ can be calculated as follows:

$$
\begin{aligned}
& \operatorname{gap}_{i}^{f}(t)=x_{i+1}^{f}(t)-x_{i}(t)-1 \\
& \operatorname{gap}_{i}^{b}(t)=x_{i}(t)-x_{i-1}^{b}(t)-1,
\end{aligned}
$$

where $x_{i-1}^{b}(t), v_{i-1}^{b}(t)$, and $v_{i-1 \max }^{b}(t)$ are the position, speed, and maximum speed of the nearest following bicycle in the lane adjacent to that of the $i$ th bicycle.

The new speed for the bicycle currently in the $i$ th position after lane-changing is calculated as follows:

$$
v_{i}^{\prime}(t+1)=\min \left[v_{i}(t)+1, \operatorname{gap}_{i}^{f}(t), v_{i \max }\right],
$$

where $v_{i}^{\prime}(t+1)$ is the speed of this bicycle after the lanechanging.

The motion of the lane-changing bicycle is

$$
x_{i}^{\prime}(t+1)=x_{i}(t)+v_{i}^{\prime}(t+1)
$$

where $x_{i}^{\prime}(t+1)$ is the position of the bicycle after the lanechanging.

2.3. M-CA Model. A family of M-CA models has recently been proposed by Nishinari and Takahashi [20-22]. The basic version of the family is obtained from an ultradiscretization of Burgers' equation. Therefore, it is also called the Burgers CA (BCA). Previously, BCA models were proposed for highway traffic. Recent attempts have included BCA models purported to represent bicycle flow $[12,13]$ adapted for the unobvious car-following and lane-changing behavior in bicycle traffic. In order to make a comparison with the NS CA model, the M-CA model for mixed bicycle flow is improved upon in this paper.

The numbers of RBs and EBs in location $j$ at time $t$ are $U_{j}^{r}(t)$ and $U_{j}^{e}(t)$, respectively. As shown in Section 2.1, RBs with a maximum speed of 2 cells/s and EBs with a maximum speed of 3 cells/s are considered in the simulation systems. Therefore, the updating procedures are changed as follows:

(1) all bicycles in location $j$ move to their next location $j+1$ if the location is not fully occupied, and EBs have priority over RBs;

(2) all bicycles that moved in procedure (1) can move to location $j+2$ if their next location is not fully occupied after procedure (1), and EBs again have priority over RBs;

(3) only EBs moved in procedure (2) can move to location $j+3$ if their next location is not fully occupied after procedure (2).

The numbers of RBs and EBs that move one location on from location $j$ at time $t$ in procedure (1) are $b_{j}^{r}(t)$ and $b_{j}^{e}(t)$, respectively. The numbers of RBs and EBs that move two locations on from location $j$ at time $t$ are $c_{j}^{r}(t)$ and $c_{j}^{e}(t)$, respectively. $d_{j}^{e}(t)$ represents the number of EBs that move three locations on from location $j$ at time $t$. $L$ is defined as the lane number of the simulation bicycle path. The randomization 
effect on the RBs is introduced as follows: $c_{j}^{r}(t+1)$ decreases by 1 with probability $p_{d r}$ if $c_{j}^{r}(t+1)>0$. The randomization effect on the EBs is as follows: $d_{j}^{e}(t+1)$ decreases by 1 with probability $p_{d e}$ if $d_{j}^{e}(t+1)>0$. The updating rules are as follows.

Step 1. Calculation of $b_{j}^{r}(t+1), b_{j}^{e}(t+1)$, and $b_{j}(t+1)(j=$ $1,2,3, \ldots, K)$ is as follows:

$$
\begin{aligned}
& b_{j}^{e}(t+1)=\min \left(U_{j}^{e}(t), L-U_{j+1}(t)\right) \\
& b_{j}^{r}(t+1)=\min \left(U_{j}^{r}(t), L-U_{j+1}(t)-b_{j}^{e}(t+1)\right) \\
& b_{j}(t+1)=b_{j}^{r}(t+1)+b_{j}^{e}(t+1) .
\end{aligned}
$$

Step 2. Calculation of $c_{j}^{r}(t+1), c_{j}^{e}(t+1)$, and $c_{j}(t+1)$ is as follows:

$$
\begin{aligned}
& c_{j}^{e}(t+1)=\min \left(b_{j}^{e}(t+1), L-U_{j+2}(t)-b_{j+1}(t+1)\right. \\
& \left.\quad+b_{j+2}(t+1)\right) \\
& c_{j}^{r}(t+1)=\min \left(b_{j}^{r}(t+1), L-U_{j+2}(t)-b_{j+1}(t+1)\right. \\
& \left.\quad+b_{j+2}(t+1)-c_{j}^{e}(t+1)\right) .
\end{aligned}
$$

If $\operatorname{rand}()<p_{d r}$, then

$$
\begin{aligned}
& c_{j}^{r}(t+1)=\max \left(c_{j}^{r}(t+1)-1,0\right) \\
& c_{j}(t+1)=c_{j}^{r}(t+1)+c_{j}^{e}(t+1) .
\end{aligned}
$$

In (12) and (15), $b_{j}^{e}(t+1)$ and $c_{j}^{e}(t+1)$ are calculated first because the EBs have priority over the RBs.

Step 3. Calculation of $d_{j}(t+1)$ is as follows:

$$
\begin{aligned}
& d_{j}(t+1)=\min \left(c_{j}^{e}(t+1), L-U_{j+3}(t)-b_{j+2}(t+1)\right. \\
& \left.+b_{j+3}(t+1)-c_{j+1}(t+1)+c_{j+2}(t+1)\right) .
\end{aligned}
$$

If $\operatorname{rand}()<p_{d e}$, then

$$
d_{j}(t+1)=\max \left(d_{j}(t+1)-1,0\right) .
$$

Step 4. Update $U_{j}^{r}(t+1), U_{j}^{e}(t+1)$, and $U_{j}(t+1)$ :

$$
\begin{aligned}
U_{j}^{e}(t+1)= & U_{j}^{e}(t)-b_{j}^{e}(t+1)+b_{j-1}^{e}(t+1) \\
& -c_{j-1}^{e}(t+1)+c_{j-2}^{e}(t+1) \\
& -d_{j-2}(t+1)+d_{j-3}(t+1) \\
U_{j}^{r}(t+1)= & U_{j}^{r}(t)-b_{j}^{r}(t+1)+b_{j-1}^{r}(t+1) \\
& -c_{j-1}^{r}(t+1)+c_{j-2}^{r}(t+1) \\
U_{j}(t+1)= & U_{j}^{r}(t+1)+U_{j}^{e}(t+1),
\end{aligned}
$$

where $\operatorname{rand}()$ is a uniformly distributed random number between 0 and 1 .

\section{Simulation Results}

For the comparison of the NS CA model against the M-CA model, the simulation parameters in both models should be set to the same values. In the simulations, a two-lane bicycle path $(L=2)$ was selected with length $K=500$ cells (equal to $1000 \mathrm{~m}$ ). In the initial conditions, RBs and EBs are randomly distributed on the road using the same random number for both models. The default values of the random slowing down probability $\left(p_{d}\right)$, the probability of a lane change $\left(p_{t}\right)$, and the proportion of EBs $\left(p_{e}\right)$ are $0.2,0.8$, and 0.5 , respectively, for the NS CA model (as in previous studies [14]). The default values of the random slowing down probability of RBs $\left(p_{d r}\right)$, the random slowing down probability of EBs $\left(p_{d e}\right)$, and the proportion of EBs are $0.4,0.4$, and 0.5 , respectively, for the $\mathrm{M}$ CA model. In the M-CA model, the slowing down probability is the probability that the number of bicycles $\left(c_{j}^{r}(t+1)\right)$ decreases, which means that one bicycle decreases its speed. In this paper, the simulation is based on two lanes $(L=2)$. Therefore, the maximum value of $c_{j}^{r}(t+1)$ is 2 . If $c_{j}^{r}(t+1)=0$, no bicycle slows down, and the slowing down probability of any bicycle is zero. If $c_{j}^{r}(t+1)=1$, only one bicycle slows down, with probability $p$. If $c_{j}^{r}(t+1)=2$, this means only one bicycle may slow down with probability $p$; therefore, the total slowing down probability of bicycles is $0.5 p$. By summing the above three cases, we assume these three cases have the same percentage. Therefore, the mean of the three cases' slowing down probabilities is $(0+p+0.5 p) / 3=0.5 p$. In order to compare the two models, we used a default value for the random slowing down probability for the M-CA model of half that for the NS CA model.

Periodic conditions that are as close as possible to the actual conditions are used so that the bicycles ride on a circuit. The instantaneous positions and speeds for all particles are updated in parallel, per second. The flow, speed, and density of the mixed bicycle traffic flow can be calculated after a given amount of time (20000 simulation steps) [15], and the averages over the last 5000 steps are used for the calculation in order to decrease the random effect.

3.1. Results of the NS CA Model. In order to show the different characteristics of the NS CA model under different model parameters, speed-density and flow-density plots (the fundamental diagram of bicycle traffic flow) were created so that the results could be analyzed. Example plots are shown in Figures 1, 2, and 3. When $p_{d}=0$, it is a deterministic case, while $p_{d} \neq 0$ is a stochastic case. From Figure 1, it can be seen that, with an increase in the slowing down probability $p_{d}$, the fundamental diagrams drop quickly, which means that the capacity of the bicycle lane drops quickly with an increase in $p_{d}$. When the slowing down probability $p_{d}$ is equal to one, the stopped RBs will lead bicycle traffic flow to jam, and the speed and flow will both be zero.

Figure 2 shows the speed-density and flow-density relationships under different lane-changing probabilities. In the low-density region, with an increase in the lane-changing probability, the speed of bicycle flow increases. In the highdensity region, the speeds of bicycle flow under different 


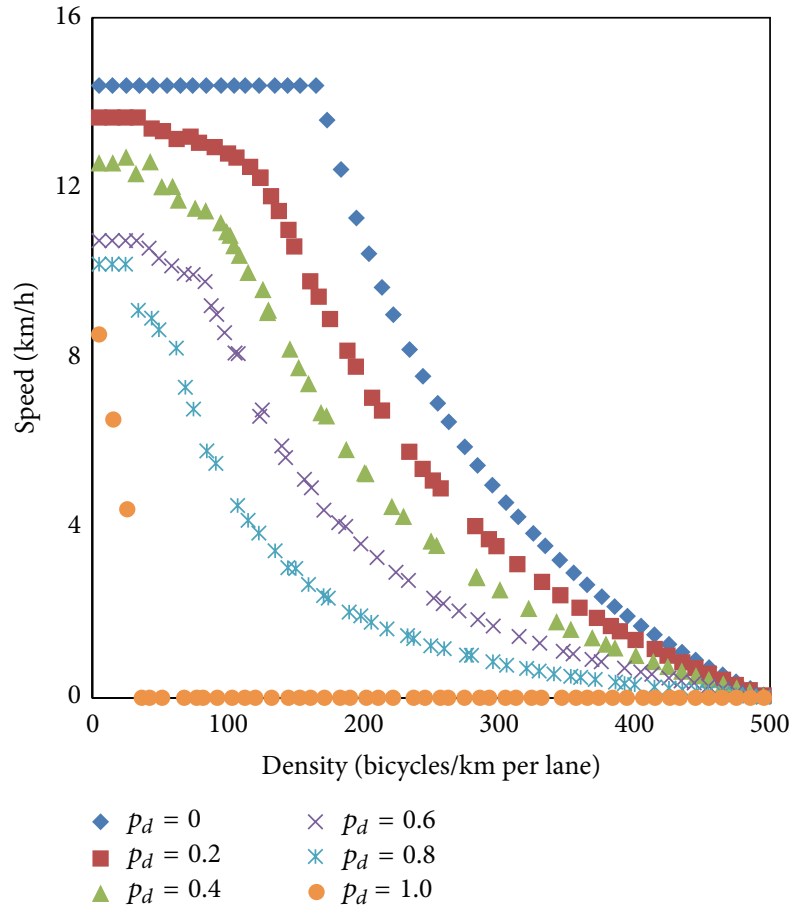

(a) Speed-density relationship

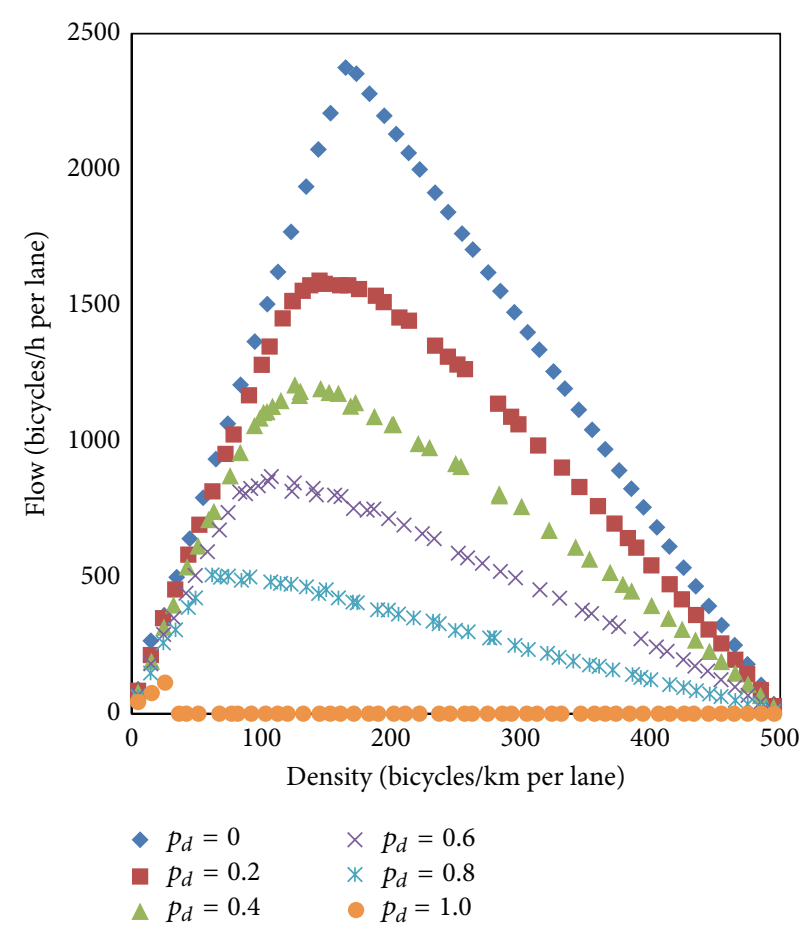

(b) Flow-density relationship

FIGURE 1: Speed-density and flow-density relationships under different slowing down probabilities when $p_{t}=0.8$ and $p_{e}=0.5$.

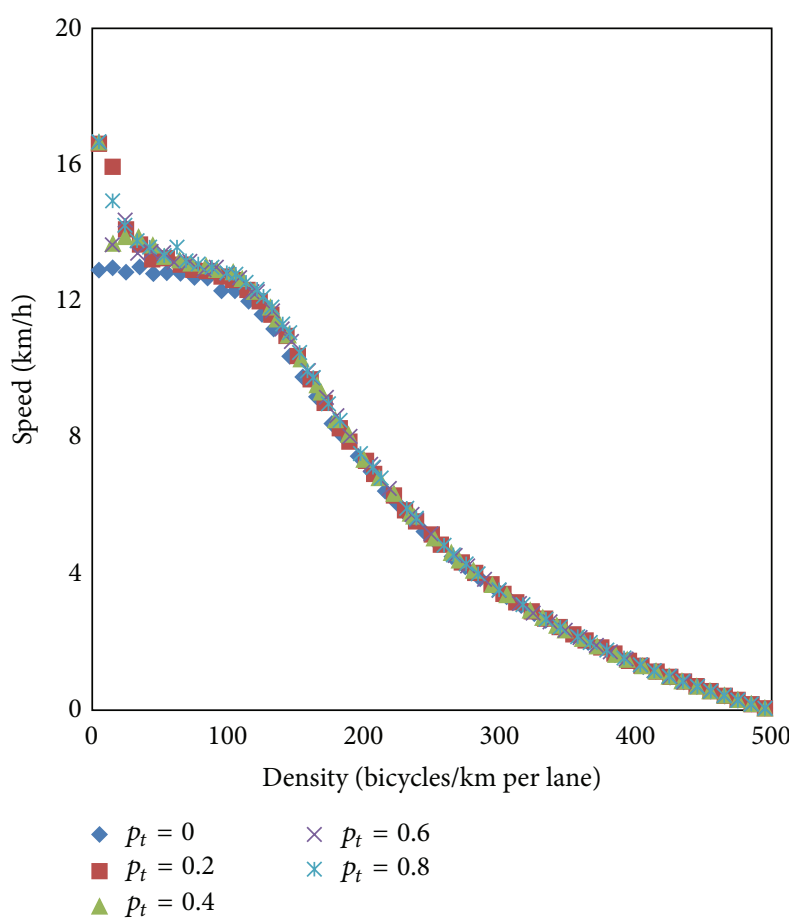

(a) Speed-density relationship

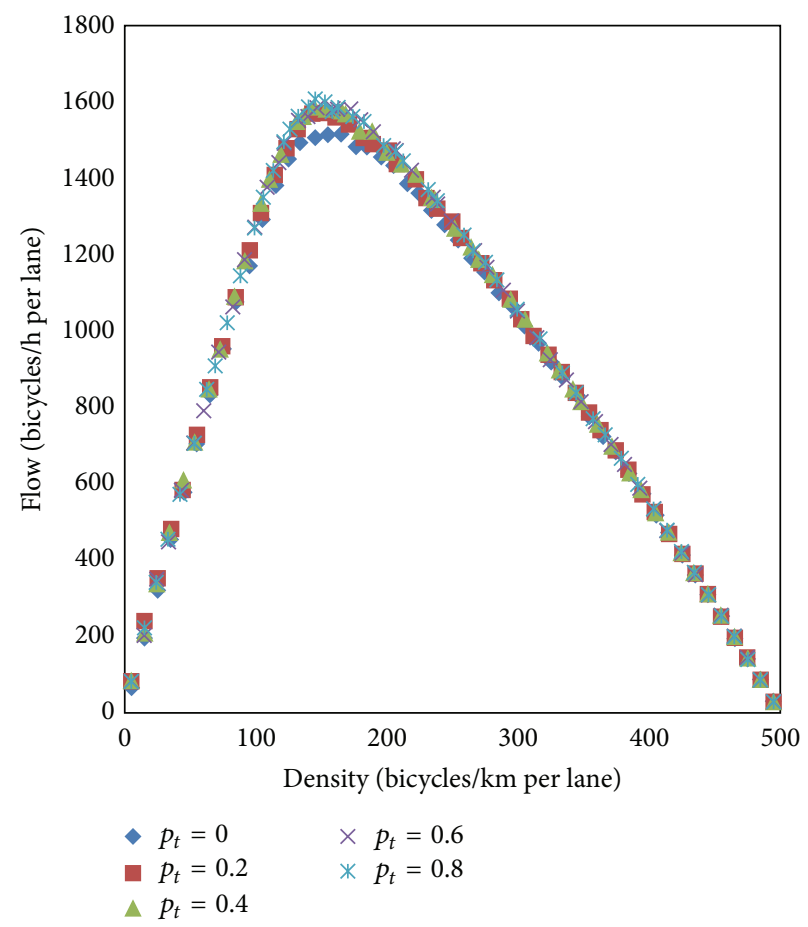

(b) Flow-density relationship

FIGURE 2: Speed-density and flow-density relationships under different lane-changing probabilities when $p_{d}=0.2$ and $p_{e}=0.5$. 


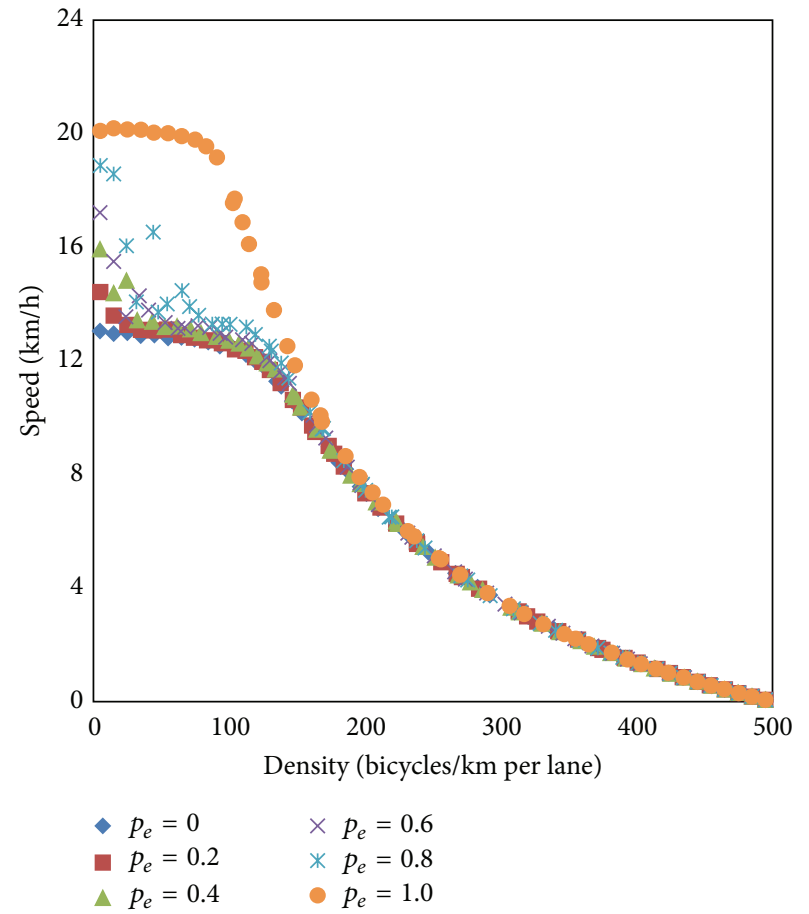

(a) Speed-density relationship

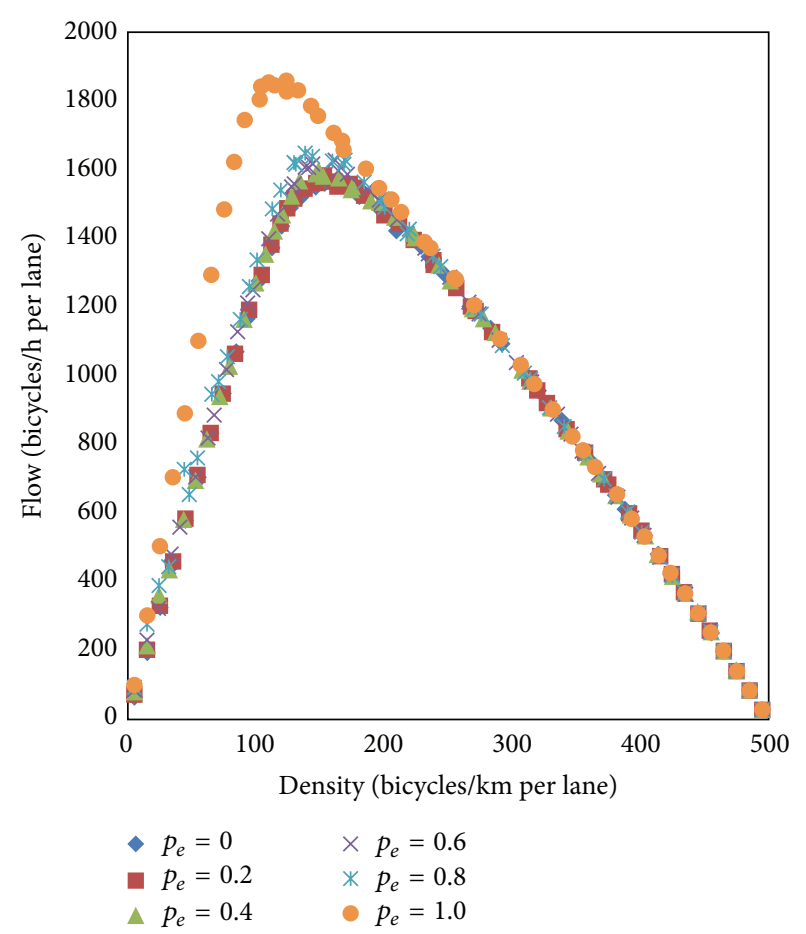

(b) Flow-density relationship

FIGURE 3: Speed-density and flow-density relationships under different proportions of EBs when $p_{d}=0.2$ and $p_{t}=0.8$.

lane-changing probabilities show smaller differences than in the low-density region. This is due to using the motorized vehicle lane-changing rule for bicycle traffic flow. The lanechanging rule proposed in this paper is very strictly based on gap acceptance theory, and the looking-backward gap should be large in order for lane-changing to happen. However, the driving behavior of bicycles is very different from that of motorized vehicles. The lane-changing rule for motorized vehicles will restrict the lane-changing behavior of higherspeed bicycles (such as EBs in this case) and lead to no significant differences between the fundamental diagrams for different lane-changing probabilities.

The proportion of EBs is one of the most important parameters for mixed bicycle traffic flow. Figure 3 shows the fundamental diagrams for different proportions of EBs in the mixed traffic. It can be seen that, with an increase in the proportion of EBs, speed and capacity increase because of the EBs' higher free flow speed compared to the RBs. Another finding observable in Figure 3 is that, when $p_{e}$ is small, the influence on capacity is small, and with the increase in $p_{e}$, the influence of $p_{e}$ on capacity becomes larger. This means that the influence of EBs on the bicycle lane capacity is not linear, which may be due to the existence of lower-speed RBs and the strict lane-changing rule, which deter EBs from changing lanes and increasing their speed.

3.2. Results of the M-CA Model. This section presents the simulation results of the M-CA model. In order to compare the results with those of the NS CA model, the slowing down probabilities of RBs and EBs are set to the same value of
0.4 . Therefore, only two parameters, $p_{d}$ and $p_{e}$, are analyzed in this simulation case. Figure 4 shows the fundamental diagrams under different $p_{d}$ values. Similarly to the NS CA model, the capacities drop with the increase in the slowing down probability. However, the capacity drops of the M-CA model are smaller than those of the NS CA model, as will be discussed in detail in the next section.

From Figure 5(a), in the low-density region (density < 200 bicycles/ $\mathrm{km}$ per lane), the bicycle flow is in the free flow state and most bicycles move independently. Therefore, the average speed of the system equals the free flow speed of mixed bicycles, which increases with the proportion of EBs. As can be seen in Figure 5(b), similar to the case of the NS CA model, with an increase in the proportion of EBs, the bicycle capacity also increases. It is easy to see that the free flow speed of all bicycles will increase with the proportion of EBs. The simulation results from the NS CA model and the M-CA model show the same findings.

\section{Discussion}

Most of the previous studies on CA models for mixed bicycle flow discuss multiple states and the transition from free flow to congested flow. However, the choice of an appropriate CA model for simulating bicycle traffic is more important than model analysis and calibration. Therefore, we should compare the simulation results of the two CA models presented above and try to draw conclusions about the selection of a CA model. 


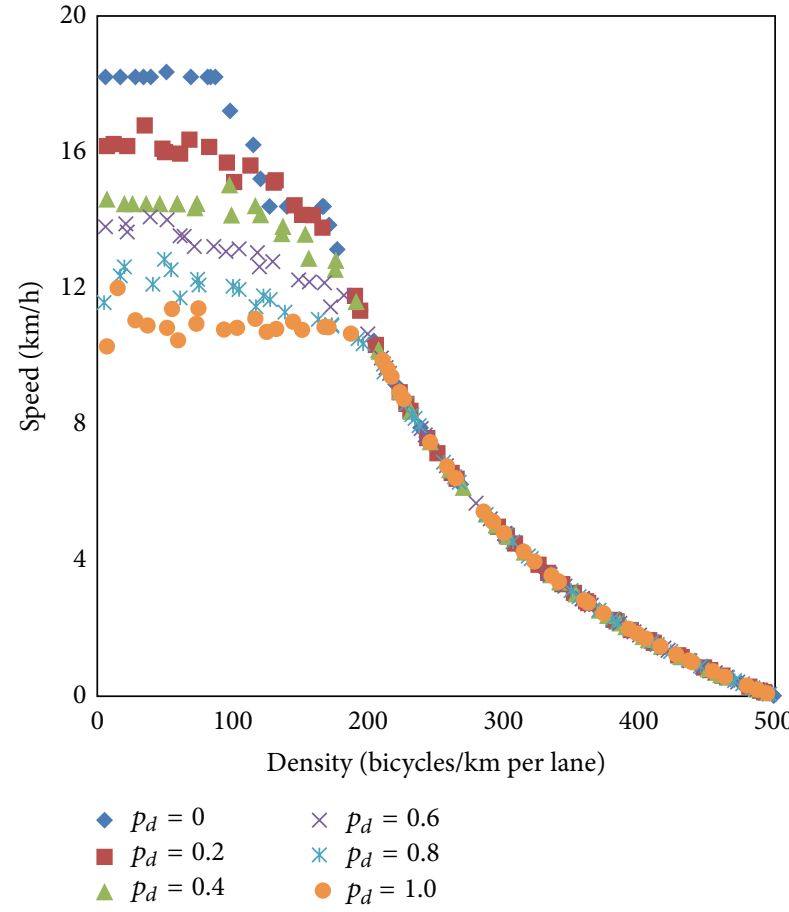

(a) Speed-density relationship

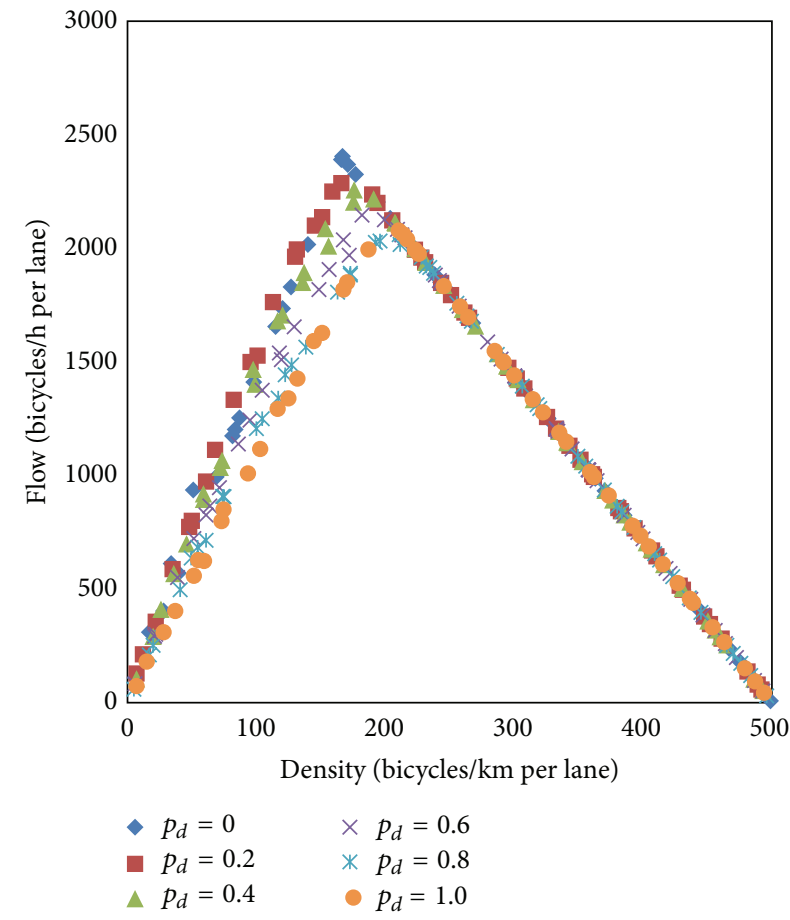

(b) Flow-density relationship

FIGURE 4: Speed-density and flow-density relationships under different slowing down probabilities of RBs and EBs when $p_{e}=0.5$.
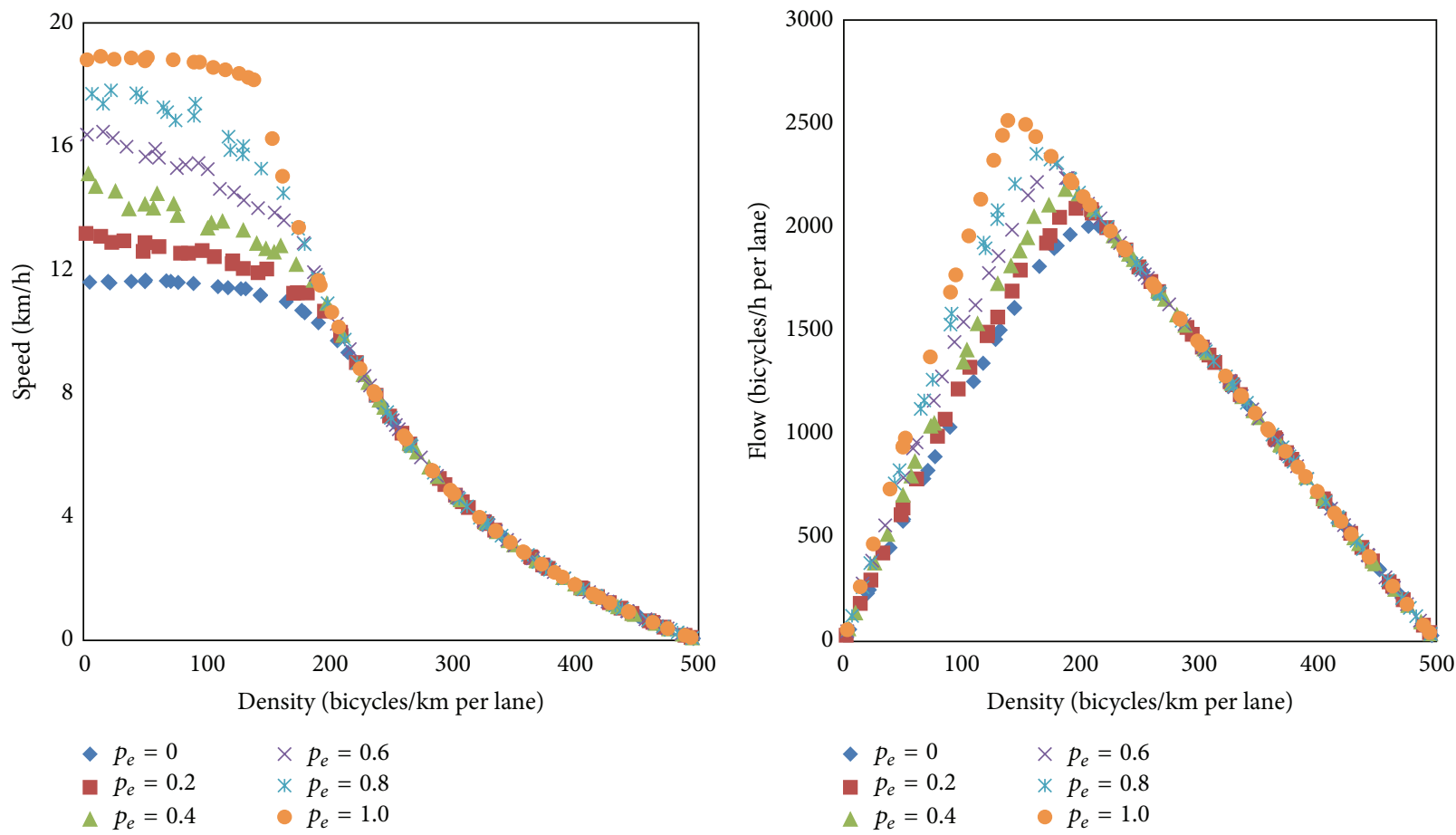
$p_{e}=0$
$p_{e}=0.2$
$\triangle p_{e}=0.4$
$\times p_{e}=0.6$
$* p_{e}=0.8$
$\times p_{e}=1.0$

(a) Speed-density relationship

(b) Flow-density relationship

FIGURE 5: Speed-density and flow-density relationships under different proportions of EBs when $p_{d}=0.4$. 


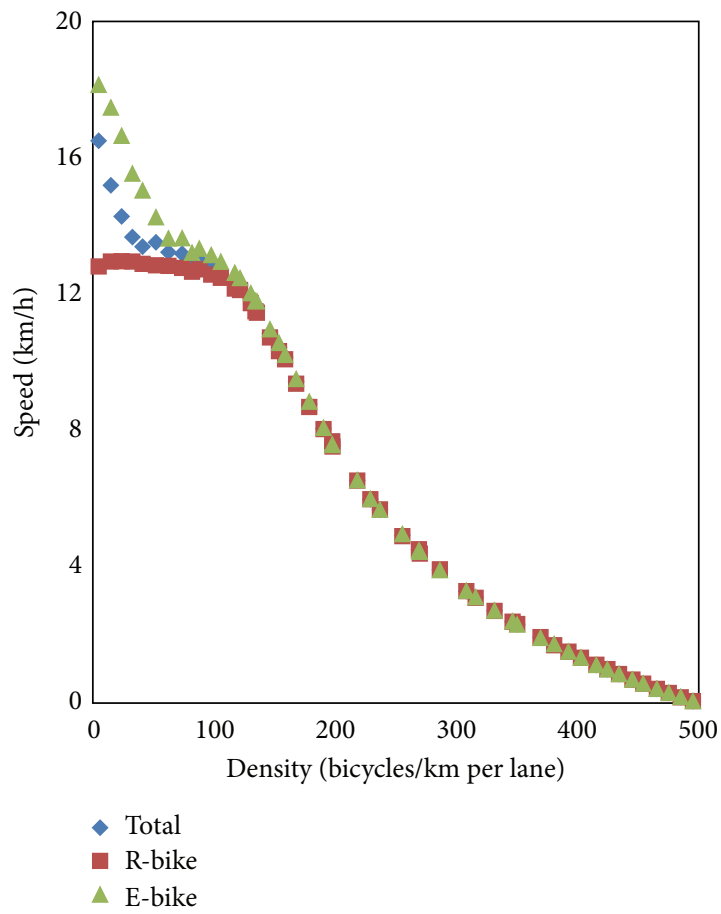

(a) NS CA model

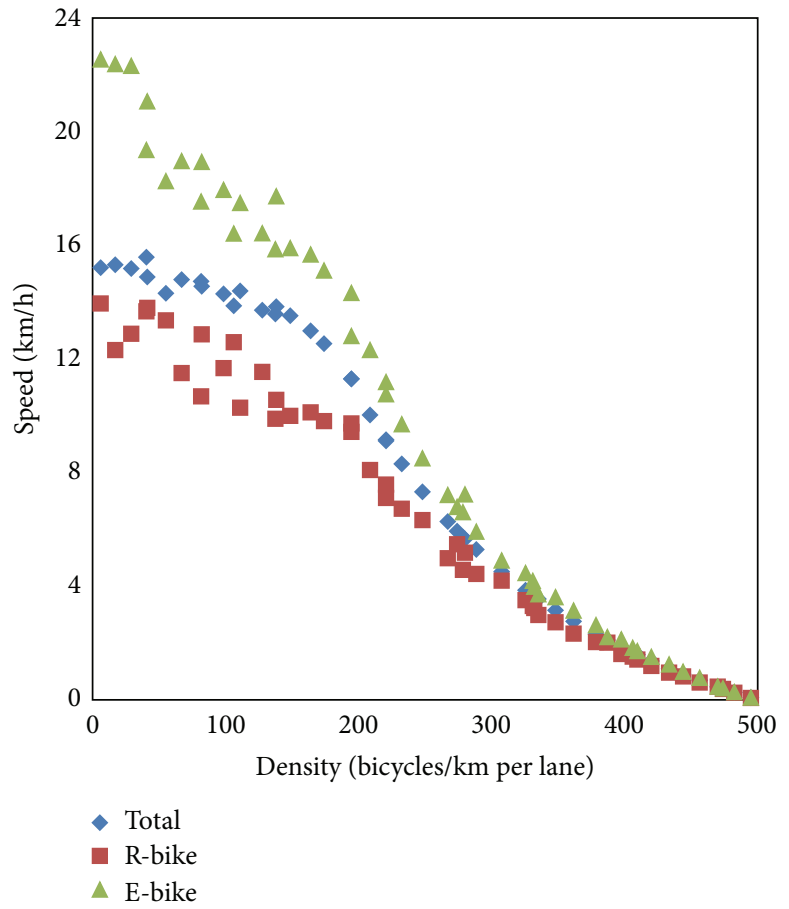

(b) M-CA model

FIGURE 6: Speed-density relationships for different bicycle types.

Because the capacity is a significant parameter for bicycle lane planning and management, capacity and fundamental diagrams can be used for evaluating the CA models [23]. The capacity is defined as the maximum flow under particular road conditions. In the fundamental diagram, the capacity is the peak of the fundamental diagram curve. For simplicity, due to the fact that the simulation densities of bicycle traffic flow cover all traffic conditions, we use the maximum flow of bicycles as the observed capacity of the bicycle path in this paper.

Figure 6 shows the comparisons of the speed-density relationships for the NS CA and M-CA models. It can easily be seen that, for the NS CA model, in the low-density region (approximately 100 bicycles $/ \mathrm{km}$ per lane or fewer), the speeds of RBs and EBs are very different, while in the high-density region the speeds of RBs and EBs are almost equal. Similar conclusions are found for the M-CA model. However, the critical density distinguishing low from high density is nearly 300 bicycles/km per lane, much larger than that for the NS CA model. The results show that the lane-changing rule of the NS CA model enables the EBs hardly to pass the RBs and the speeds of both bicycle types to quickly become the same.

Figure 7 shows the simulated capacity values obtained from the maximum values of the fundamental diagrams. It can be seen that when the slowing down probability is zero (meaning that both models are deterministic CA models) the two CA models have the same capacity (NS CA model 2387 bicycles/h per lane; M-CA model 2375 bicycles/h per lane). With the increase in the slowing down probability, the capacities of both models drop linearly. Linear regression equations are also shown in Figure 7, and it can clearly be seen that there are strongly linear relationships between the capacities of the two models and the slowing down probabilities. However, the difference between the regression model slopes of the two models is large $(-407.27$ versus -2045.3). Therefore, when the slowing down probability of the M-CA model equals 1 , which means that the slowing down probability of the NS CA model is nearly 0.5 , the maximum volumes of the two models are very different $(2000$ versus 1000 bicycles/h per lane). This means that the slowing down probability has a greater influence on the NS CA model than the M-CA model.

The slowing down probability, as the most significant parameter of the CA model, describes the stochastic effects on bicycle traffic flow. Because of the strict lane-changing rule in the NS CA model, the EBs (fast bicycles) do not find it easy to change lanes and must follow the RBs, which leads to a very low capacity. However, the M-CA model has an implied lanechanging rule in the update rules which has less influence on capacity than in the NS CA model.

Field bicycle data were collected at Jiaogong Road in Hangzhou, China. The width of this bicycle path is $2.27 \mathrm{~m}$, making it nearly a two-lane bicycle path. The field data cover all traffic conditions, and the EB percentages also cover a wide range. The average percentage of EBs is $60.3 \%$, and the capacity of the bicycle path is defined as the maximum volume, with a 30 -second sampling interval. Figure 8 shows the observed and simulated speed-density and flow-density relationships, where the percentage of EBs is set to 0.6 (equal to the field sample percentage) and other simulation 


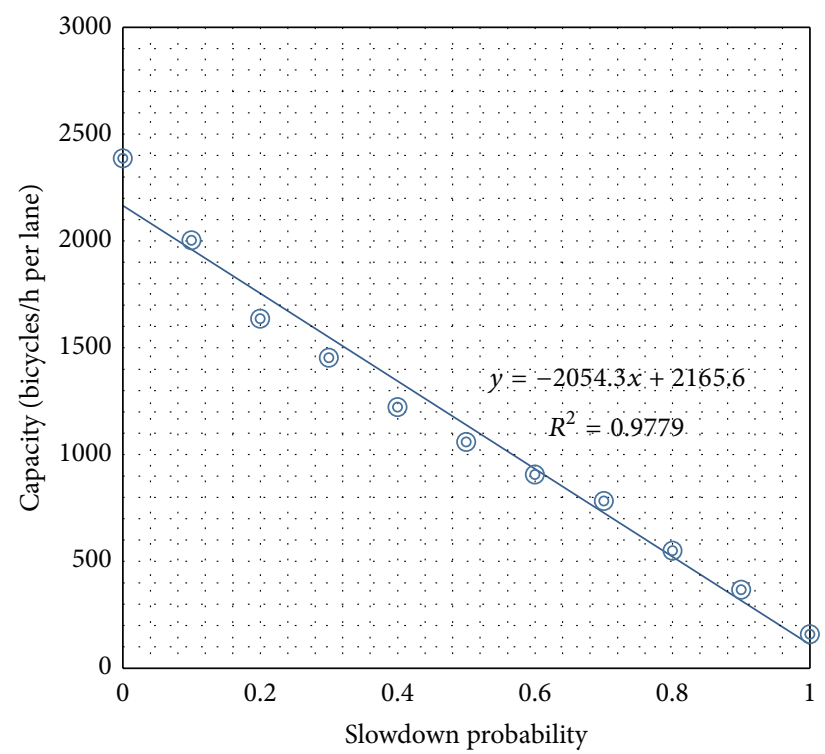

(a) NS CA model

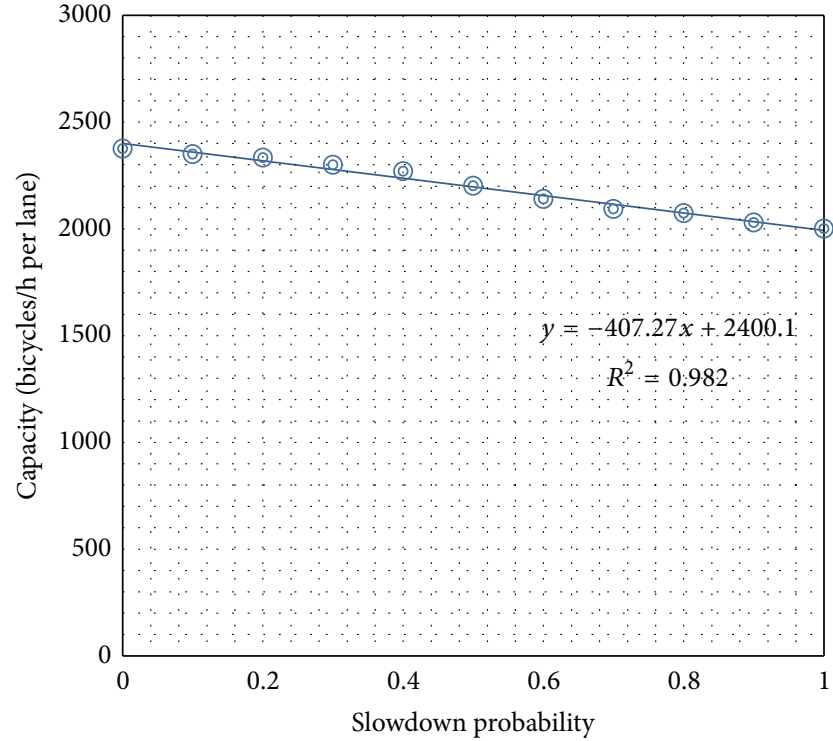

(b) M-CA model

FIGURE 7: Simulated capacities of two CA models under different slowing down probabilities.

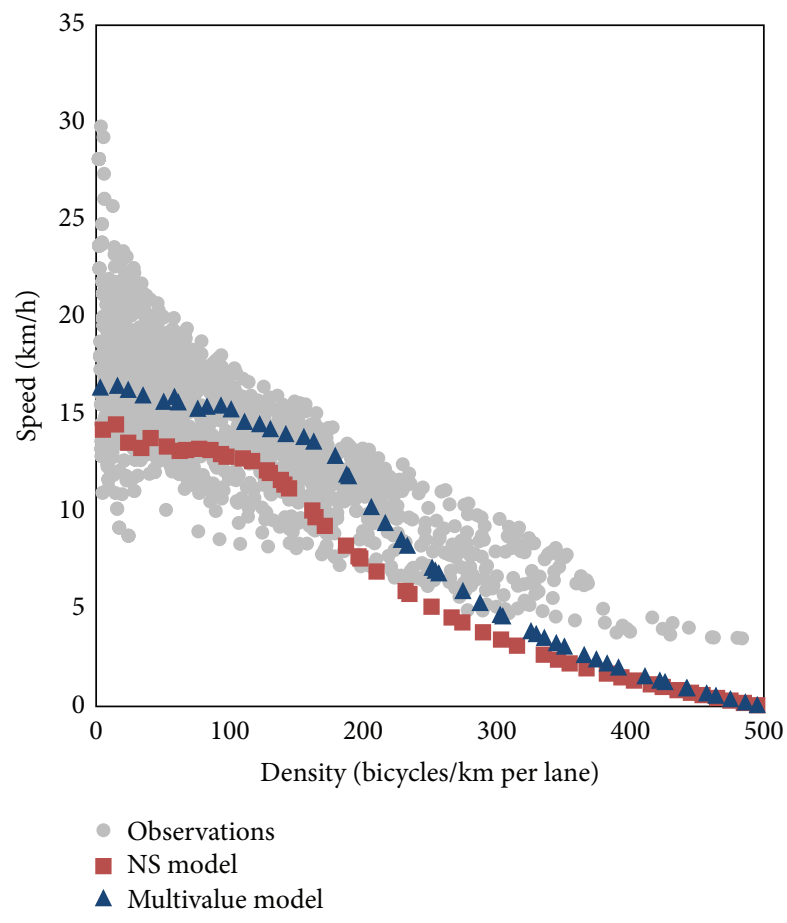

(a) Speed-density relationship

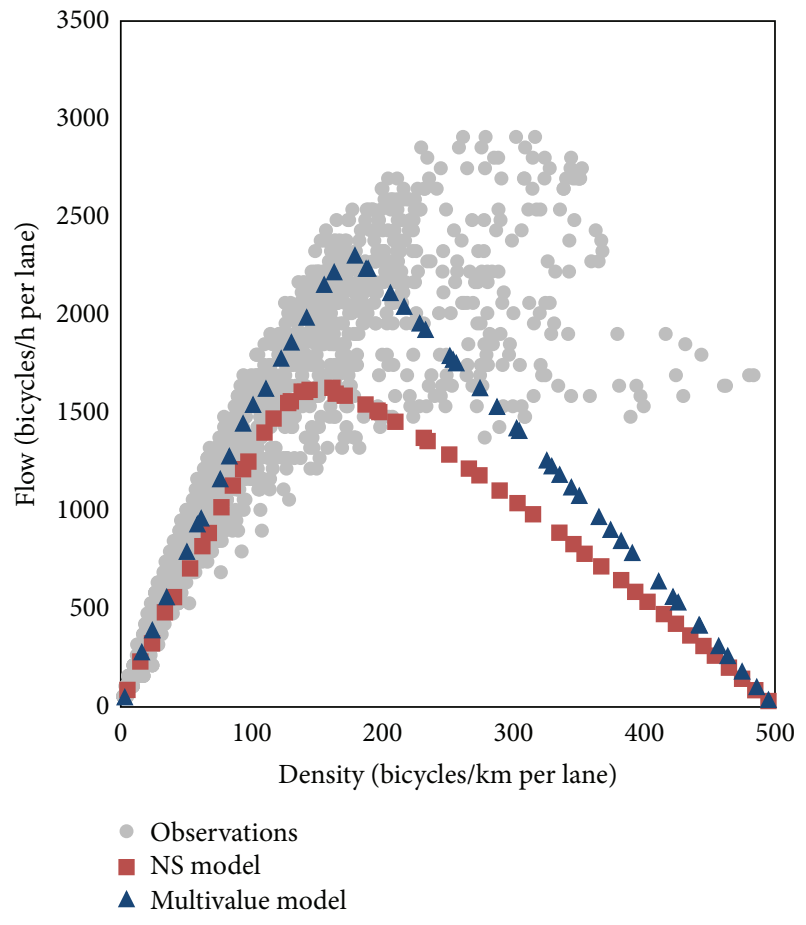

(b) Flow-density relationship

FIGURE 8: Observed and simulated speed-density and flow-density relationships.

parameters are set to the default values presented above. The results imply that the M-CA model performs better than the NS model in fitting the field bicycle observations.

The proportion of EBs describes the proportion of fast bicycles in the mixed bicycle traffic, which affects the free flow speed and the capacity of the bicycle lane. Figure 9 shows the relationships between the proportion of EBs and the lane capacity from observed and simulated results. It can be seen that both models produce the same nonlinear relationship, and the correlation coefficients are both very high. When the proportion of EBs is low, the EBs must slow down their speed and follow the RBs. Therefore, the capacity slowly increases 


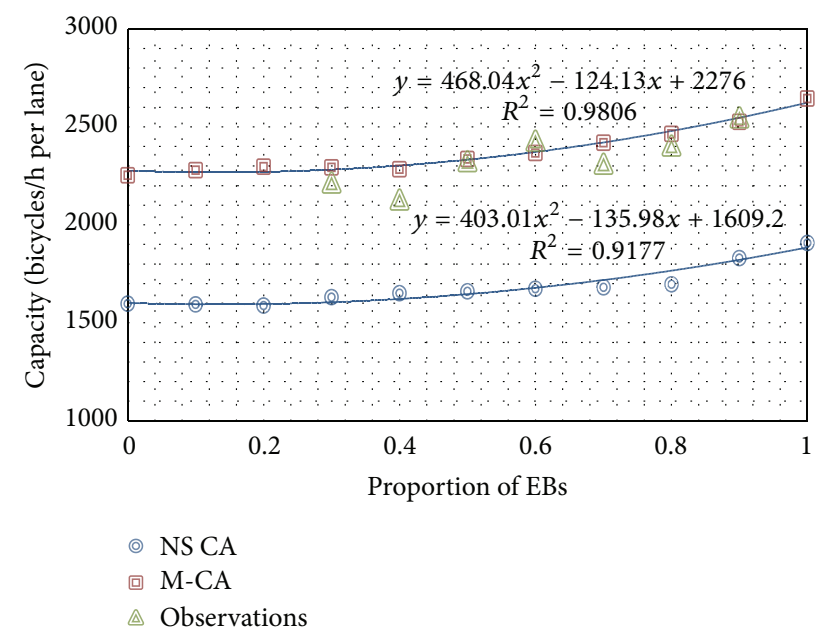

FIGURE 9: Observed and simulated capacities under different proportions of EBs.

with the proportion of EBs. When the proportion of EBs is large, lane-changing and passing occur more frequently, and the capacity increases quickly with the proportion of EBs. The simulated results of the M-CA model seem more consistent with the observed field bicycle capacity than those of the NS CA model. The root mean square error (RMSE) and the mean absolute percentage error (MAPE) $[24,25]$ of M-CA model are less than those of NS CA model.

Based on the above comparison and analysis, some conclusions can be drawn. Firstly, CA models can be used for bicycle traffic simulation because of their simple rules and quick simulation. The results for the fundamental diagrams and capacities (about 2000-2500 bicycles/h per lane) are similar to those from the field data and previous studies [26]. Secondly, the slowing down probability has a significant influence on the simulation results for both the NS CA model and the M-CA model. Meanwhile, with an increase in the slowing down probability, the capacity and speed drop more quickly in the NS CA model than in the M-CA model. This may be due to the lane-changing rule of NS CA models that restricts EBs in changing lanes and accelerating. Thirdly, the proportion of EBs in the mixed traffic flow affects the critical density and capacity in both the NS CA model and the M-CA model, as was also reported by some researchers [27, 28]. As the proportion of EBs moves from 0 to 1, the capacities of the NS CA model and the M-CA model increase $19.3 \%$ and $17.4 \%$, respectively. Fourthly, for the NS CA model, the probability of lane-changing has less influence on the mixed traffic flow than does the slowing down probability, which may be due to the strict lane-changing rule leading to less lane-changing by bicycles. Lastly, the NS CA model is restricted for multilane bicycle path simulation because more bicycle lanes will lead to more complicated lane-changing rules that are hard to model and calibrate. In contrast, with the M-CA model it is easy to simulate a multilane bicycle path, by simply setting different $L$ values. As can be seen from the above summary, the MCA model provides more effective performance for modeling bicycle traffic and is more consistent with the field bicycle data than the NS CA model.

\section{Conclusions}

The modeling and simulation of mixed bicycle traffic flow are becoming increasingly significant because of the increased popularity of regular bicycles and electric bicycles in recent years, due to their greenness and convenience. This paper has proposed two improved CA models for bicycle traffic flow modeling and simulation and has compared their characteristics. The two-lane NS CA model and the multivalue CA model for mixed bicycle traffic flow were introduced and the same parameters set for both models so that a comparison could be made under the same conditions. Speed-density and flow-density relations were obtained so as to compare and analyze the models, and the capacities obtained from the simulation results were also compared under different model parameters. Field data collected from Hangzhou, China, were used for the evaluation of the proposed models. The results show that the M-CA model performs better than the NS CA model in simulating mixed bicycle traffic. The main difference between these two models is the lane-changing and slowing down probability rules, making it harder or easier for EBs to change lanes and accelerate to their free flow speed.

Because of the difficulty of collecting bicycle field data, especially in congested traffic conditions, the calibration and validation of the proposed model using field data were omitted from this paper, and only simulation results were analyzed and compared between the two models. Future work will focus on the calibration of the slowing down probabilities and lane-changing probabilities under different traffic conditions, so as to further validate and evaluate the proposed models.

\section{Conflict of Interests}

The authors declare that there is no conflict of commercial or associative interests regarding the publication of this work.

\section{Acknowledgments}

This work was supported by the National Natural Science Foundation of China (nos. 51338008, 51278454, 51208462, and 61304191), the Fundamental Research Funds for the Central Universities (2014QNA4018), the Projects in the National Science \& Technology Pillar Program (2014BAG03B05), and the Key Science and Technology Innovation Team of Zhejiang Province (2013TD09).

\section{References}

[1] A. D. May, Traffic Flow Fundamentals, Prentice-Hall, Englewood Cliffs, NJ, USA, 1990.

[2] D. C. Gazis, R. Herman, and R. W. Rothery, "Nonlinear followthe-leader models of traffic flow," Operations Research, vol. 9, no. 4, pp. 545-567, 1961. 
[3] S. Jin, D.-H. Wang, Z.-Y. Huang, and P.-F. Tao, "Visual angle model for car-following theory," Physica A: Statistical Mechanics and Its Applications, vol. 390, no. 11, pp. 1931-1940, 2011.

[4] S. Jin, D.-H. Wang, and X.-R. Yang, "Non-lane-based car-following model with visual angle information," Transportation Research Record: Journal of the Transportation Research Board, no. 2249, pp. 7-14, 2011.

[5] S. Jin, D.-H. Wang, C. Xu, and Z.-Y. Huang, "Staggered carfollowing induced by lateral separation effects in traffic flow," Physics Letters A, vol. 376, no. 3, pp. 153-157, 2012.

[6] M. Brackstone and M. McDonald, "Car-following: a historical review," Transportation Research F: Traffic Psychology and Behaviour, vol. 2, no. 4, pp. 181-196, 1999.

[7] D. Chowdhury, L. Santen, and A. Schadschneider, "Statistical physics of vehicular traffic and some related systems," Physics Reports, vol. 329, no. 4-6, pp. 199-329, 2000.

[8] S. Wolfram, Theory and Applications of Cellular Automata, Advanced Series on Complex Systems, World Scientific, Singapore, 1986.

[9] K. Nagel and M. Schreckenberg, "A cellular automaton model for freeway traffic," Journal de Physique I France, vol. 2, no. 12, pp. 2221-2229, 1992.

[10] R. Jiang, B. Jia, and Q.-S. Wu, "Stochastic multi-value cellular automata models for bicycle flow," Journal of Physics A: Mathematical and General, vol. 37, no. 6, pp. 2063-2072, 2004.

[11] L. W. Lan and C.-W. Chang, "Inhomogeneous cellular automata modeling for mixed traffic with cars and motorcycles," Journal of Advanced Transportation, vol. 39, no. 3, pp. 323-349, 2005.

[12] B. Jia, X.-G. Li, R. Jiang, and Z.-Y. Gao, "Multi-value cellular automata model for mixed bicycle flow," The European Physical Journal B, vol. 56, no. 3, pp. 247-252, 2007.

[13] X.-G. Li, Z.-Y. Gao, X.-M. Zhao, and B. Jia, "Multi-value cellular automata model for mixed non-motorized traffic flow," Acta Physica Sinica, vol. 57, no. 8, pp. 4777-4785, 2008.

[14] G. Gould and A. Karner, "Modeling bicycle facility operation: cellular automaton approach," Transportation Research Record, no. 2140, pp. 157-164, 2009.

[15] X.-F. Yang, Z.-Y. Niu, and J.-R. Wang, "Mixed non-motorized traffic flow capacity based on multi-value cellular automata model," Journal of System Simulation, vol. 24, no. 12, pp. 25772581, 2012.

[16] S. Zhang, G. Ren, and R. Yang, "Simulation model of speeddensity characteristics for mixed bicycle flow-comparison between cellular automata model and gas dynamics model," Physica A: Statistical Mechanics and Its Applications, vol. 392, no. 20, pp. 5110-5118, 2013.

[17] Ministry of Housing and Urban-Rural Development of China (MOHURD), "Code for design of urban road engineering," Tech. Rep. CJJ37-2012, MOHURD, 2012.

[18] Transportation Research Board, Highway Capacity Manual (2010), National Research Council, Washington, DC, USA, 2010.

[19] M. Rickert, K. Nagel, M. Schreckenberg, and A. Latour, "Two lane traffic simulations using cellular automata," Physica A, vol. 231, no. 4, pp. 534-550, 1996.

[20] K. Nishinari and D. Takahashi, "Analytical properties of ultradiscrete Burgers equation and rule-184 cellular automaton," Journal of Physics A, vol. 31, no. 24, pp. 5439-5450, 1998.

[21] K. Nishinari and D. Takahashi, "A new deterministic CA model for traffic flow with multiple states," Journal of Physics A, vol. 32, no. 1, pp. 93-104, 1999.
[22] K. Nishinari and D. Takahashi, "Multi-value cellular automaton models and metastable states in a congested phase," Journal of Physics A: Mathematical and General, vol. 33, no. 43, pp. 77097720, 2000.

[23] X. Qu, S. Wang, and J. Zhang, "On the fundamental diagram for freeway traffic: a novel calibration approach for single-regime models," Transportation Research Part B: Methodological, vol. 73, pp. 91-102, 2015.

[24] S. Jin, D.-H. Wang, C. Xu, and D.-F. Ma, "Short-term traffic safety forecasting using Gaussian mixture model and Kalman filter," Journal of Zhejiang University SCIENCE A, vol. 14, no. 4, pp. 231-243, 2013.

[25] Y. Kuang, X. Qu, and S. Wang, "A tree-structured crash surrogate measure for freeways," Accident Analysis \& Prevention, vol. 77, pp. 137-148, 2015.

[26] S. Jin, X. Qu, D. Zhou, C. Xu, D. Ma, and D. Wang, "Estimating cycleway capacity and bicycle equivalent unit for electric bicycles," Transportation Research Part A, vol. 77, pp. 225-248, 2015.

[27] D. Wang, D. Zhou, S. Jin, and D. Ma, "Characteristics of mixed bicycle traffic flow on conventional bicycle path," in Proceedings of the 94th Annual Meeting of the Transportation Research Board, Washington, DC, USA, 2015.

[28] D. Zhou, C. Xu, D. Wang, and S. Jin, "Estimating capacity of bicycle path on urban roads in Hangzhou, China," in Proceedings of the 94th Annual Meeting of the Transportation Research Board, Washington, DC, USA, 2015. 


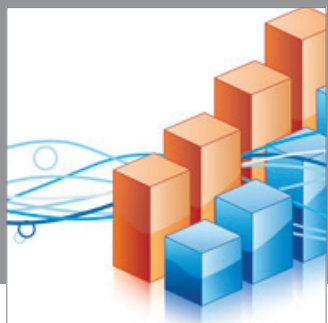

Advances in

Operations Research

mansans

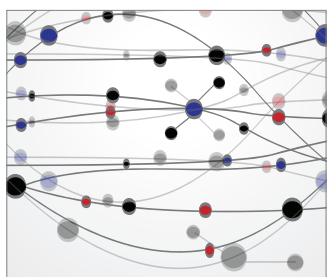

The Scientific World Journal
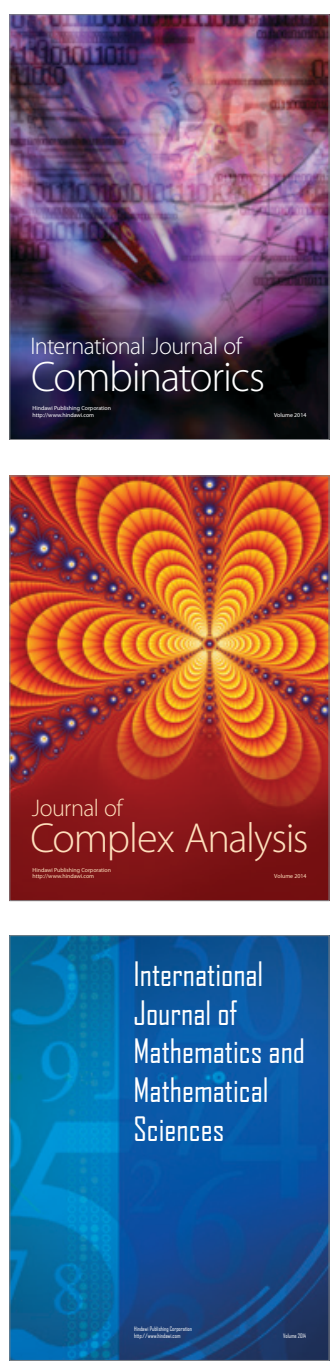
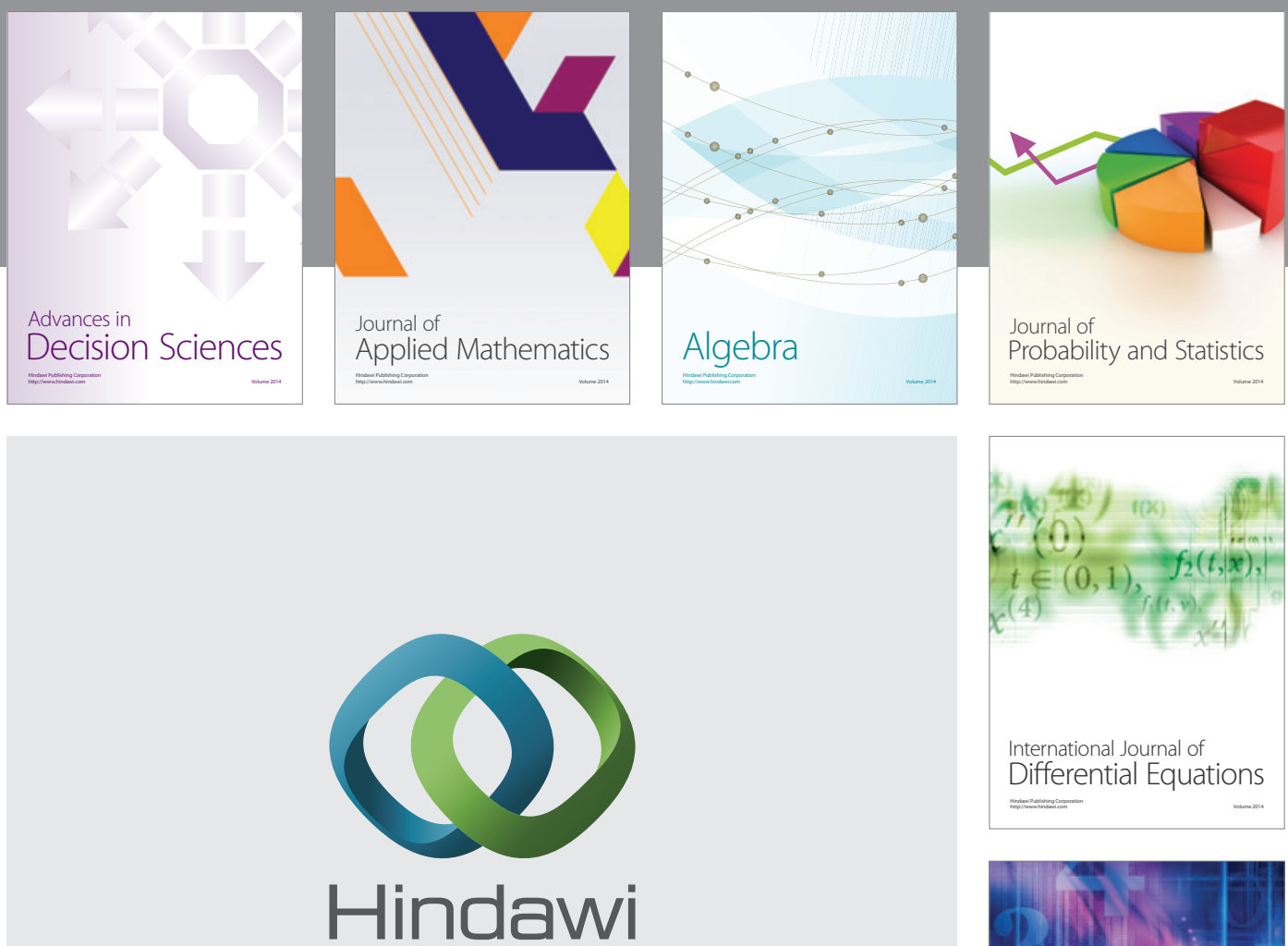

Submit your manuscripts at http://www.hindawi.com
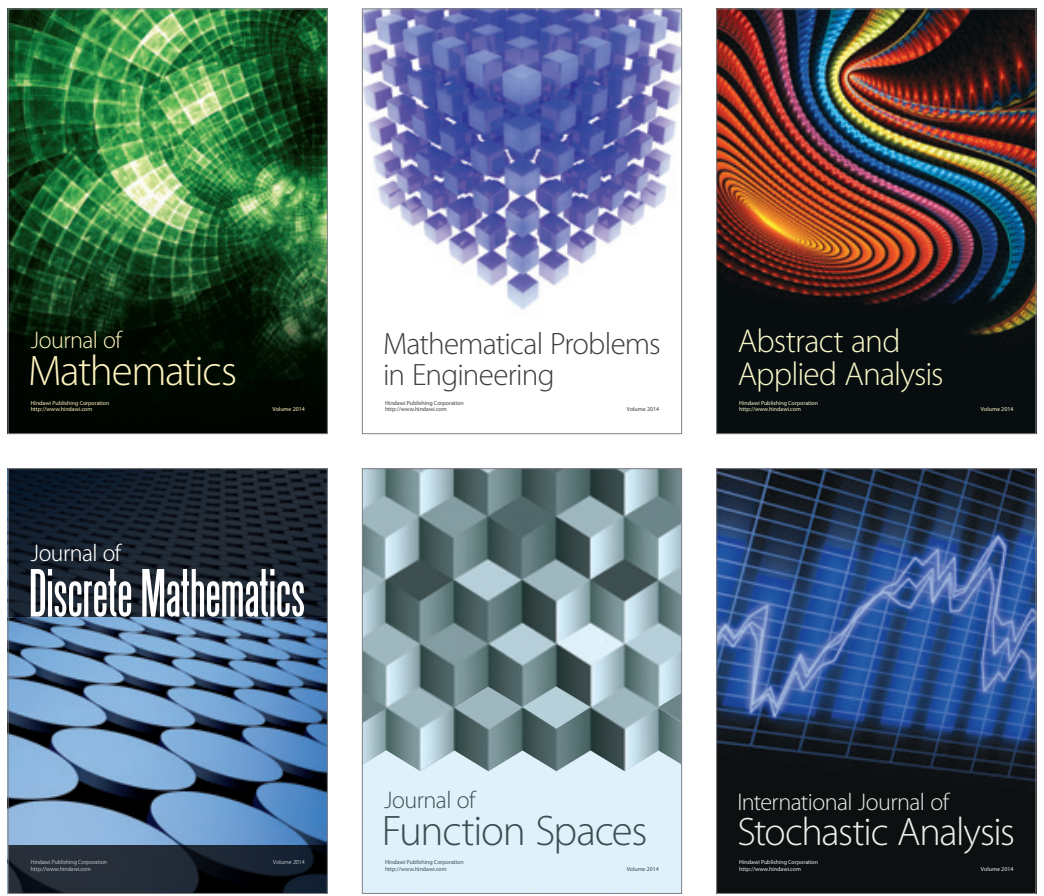

Journal of

Function Spaces

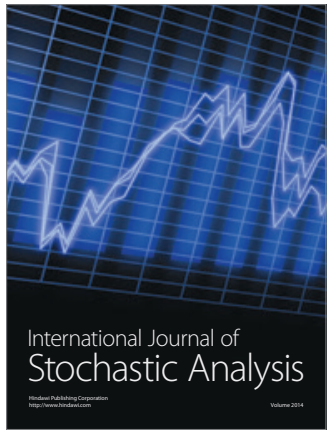

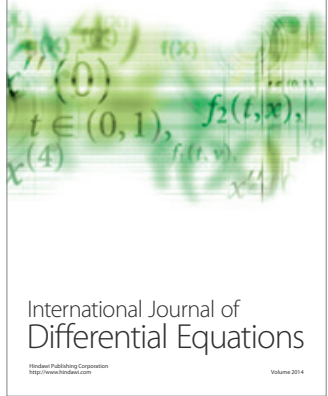
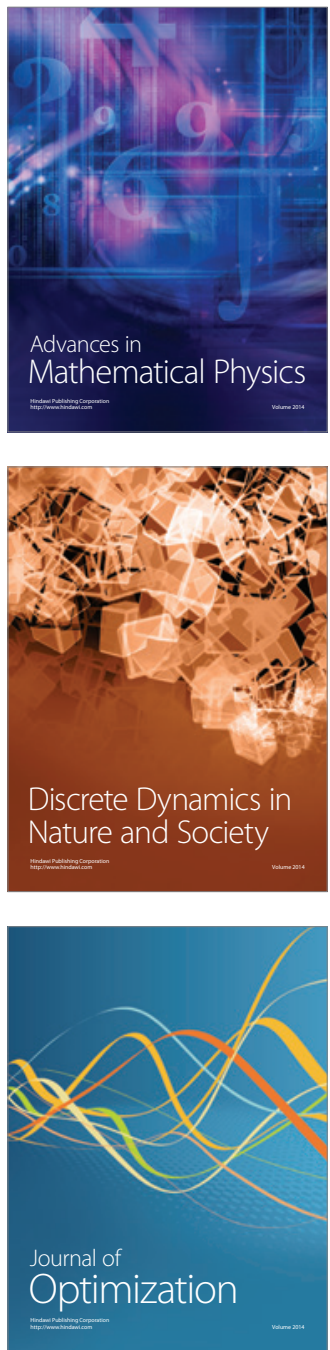